\title{
NOVIKOV CONJECTURES AND RELATIVE HYPERBOLICITY
}

\author{
BORIS GOLDFARB
}

\begin{abstract}
We consider a class of relatively hyperbolic groups in the sense of Gromov and use an argument modeled after Carlsson-Pedersen to prove Novikov conjectures for these groups. This proof is related to $[16,17]$ which dealt with arithmetic lattices in rank one symmetric spaces and some other arithmetic groups of higher rank. Here we view the rank one lattices in this different larger context of relative hyperbolicity which also includes fundamental groups of pinched hyperbolic manifolds. Another large family of groups from this class is produced using combinatorial hyperbolization techniques.
\end{abstract}

\section{Introduction}

This paper is concerned with an application of algebraic $K$-theory to questions in geometric topology. We study the assembly map which can be conjecturally used to assemble the $K$-theory of a group ring from the information about the classifying space of the group and the $K$-theory of the ring. There is at least a couple of reasons why this algebraic map is of importance in topology. One is the involvement of the $K$-theory of the integral group ring $Z G$ in the description of the space of automorphisms of a manifold with fundamental group $G$. The other is the connection with Novikov and Borel conjectures.

It is known that the homotopy invariance of higher signatures conjectured by S.P. Novikov follows from the splitting of the rational assembly map in $L$-theory. This assembly naturally maps the rational group homology containing the signature to the surgery $L$-group where the image is a priori homotopy invariant. If the assembly is actually a split injection then the signature is homotopy invariant. This is the modern approach to proving the conjecture which is believed to be true for all torsion-free groups $G$. In fact, stronger integral conjectures can be stated when integral group homology is used, and there are $K$-, $A$-theoretic, and $C^{*}$-algebraic analogues of these integral maps. So by the integral Novikov conjecture for a discrete group $G$ in

Received January 9, 1997; in revised form March 11, 1997. 
$S$-theory we understand the statement that the assembly map $\alpha(G): B G_{+} \wedge S(R) \rightarrow S(R G)$ is a split injection, where $S$ denotes the corresponding $S$-theory spectrum. The approach to these conjectures initiated in [4] and developed further in [5, 6, 7, 8, 9] can be made to apply in each of these situations. For simplicity, we will consider only the assembly map in algebraic $K$-theory. We will show

MaIn THeORem. If $G$ is a relatively hyperbolic group satisfying two assumptions (2.3) and (3.4) below then the assembly map $\alpha(G)$ is a split injection.

Of the two assumptions in the statement, (2.3) is rather standard in the recent work on Novikov conjectures and (3.4) is a certain induction hypothesis special to our geometric situation.

The prime examples of this situation are torsion-free fundamental groups of pinched hyperbolic manifolds. It is known from Gromov-Thurston [19] that this class is already larger than the rank one lattices. The hyperbolization techniques of Gromov-Charney-Davis-Januszkiewicz produce many other examples which justify our use of metric, non-differential geometry.

Section 2 introduces relatively hyperbolic groups, formulates Assumption 2.3, and establishes some geometric properties. Section 3 sketches the method from [17] and formulates Assumption 3.4. Section 4 compactifies $E G$ for a relatively hyperbolic group $G$ satisfying our assumptions. Section 5 completes the proof of the Main Theorem and demonstrates examples of groups to which our theorem applies.

ACKNOWLedgement. I would like to thank Jim West for many discussions we had related to the subject of this paper.

\section{Relatively Hyperbolic Groups}

In his seminal article [18] on hyperbolic groups, M. Gromov considered certain groups he called hyperbolic relative to a given system of subgroups $(\S 0.2(\mathrm{~F}))$ in the context of his "non-definition" of semi-hyperbolicity $(\S 0.2(\mathrm{E}))$ and concluded his paper with $\S 8.6$ studying them. The notion of semi-hyperbolicity has been made precise later by Alonso-Bridson [1], and Farb [14] defined relative hyperbolicity in the way which extends naturally to automatic groups. (There seems to be no direct relation between these notions any longer.) We will be using the term relatively hyperbolic groups in the original sense of Gromov. They are not semi-hyperbolic in the sense of Alonso-Bridson but are relatively hyperbolic in the sense of Farb.

Geodesic Metric Spaces. Let $(X, d)$ be a metric space. A geodesic segment between two points $x$ and $y$ in $X$ is an isometry $\phi:[0, d(x, y)] \rightarrow X$ with 
$\phi(0)=x, \phi(d(x, y))=y$. A metric space $X$ is called geodesic if every two points in $X$ can be joined by a segment. It is complete if every geodesic segment in $X$ can be extended to $[0, \infty)$ so that each $\phi \mid[0, l]$ is a geodesic segment.

A geodesic metric space $X$ is $\delta$-hyperbolic or simply hyperbolic if there exists $\delta>0$ such that geodesic triangles in $X$ are uniformly $\delta$-thin, i.e., the $\delta$ neighborhood of any two sides of a geodesic triangle contains the other side.

Given a real number $\kappa$, let $M_{\kappa}$ be the complete simply connected Riemannian surface of constant curvature $\kappa$. For any geodesic triangle $\Delta$ with vertices $p, q, r$ in a geodesic metric space $(X, d)$, there is a comparison triangle $\bar{\Delta}$ in $M_{\kappa}$ with vertices $\bar{p}, \bar{q}$, and $\bar{r}$, so that the lengths of the corresponding sides are $d(p, q), d(q, r), d(r, p)$. Given a side of $\Delta$ and a point $x$ on it, let $\bar{x}$ be the comparison point dividing the corresponding side of $\bar{\Delta}$ in the same proportion. The space $X$ is a $\operatorname{CAT}(\kappa)$ space if $d(x, y) \leq d(\bar{x}, \bar{y})$ for each geodesic triangle $\Delta$ in $X$ and each pair of points $x$ and $y$ on the sides of $\Delta$. It is known that all $\operatorname{CAT}(\kappa)$ spaces for $\kappa<0$ are hyperbolic and that $\operatorname{CAT}\left(\kappa_{1}\right)$ implies $\operatorname{CAT}\left(\kappa_{2}\right)$ for all $\kappa_{1}<\kappa_{2} \leq 0$.

Each hyperbolic or $\operatorname{CAT}(0)$ space $X$ has the ideal boundary $\partial X$, and there is a way to topologize $\varepsilon X=X \cup \partial X$ so that it becomes a compact metrizable space with the action of self-isometries of $X$ extending to $\partial X$ (see [15, Ch. 7]).

Hyperbolic and Relatively Hyperbolic Groups. Let $G$ be a discrete group with finite generating set $\Sigma$. The Cayley graph is a 1-complex with vertices $V(G, \Sigma)=G$ and edges $E(G, \Sigma)=\{\{g, g \sigma\}: g \in G, \sigma \in \Sigma\}$. Let each edge in $\mathrm{Ca}(G, \Sigma)$ have unit length. This induces a path-metric on $\mathrm{Ca}(G, \Sigma)$.

2.1. Definition. A discrete group $G$ is hyperbolic in the sense of Gromov if its Cayley graph is hyperbolic. This property is independent of the choice of $\Sigma$.

Let $X$ be a complete locally compact CAT $(\kappa)$ space for some $\kappa<0$ and $G$ be a discrete group of free isometries of $X$ with the quotient $V=X / G$ quasiisometric to the wedge of $k$ copies of rays $[0, \infty)$ with the standard metric. There is a quasi-isometric embedding of this wedge in $V$ with each ray $R_{i}:[0, \infty) \rightarrow V$ representing a point $\partial R_{i} \in \partial V$. Choose a lift $x_{0} \in X$ of the wedge point in $V$ and lifts $r_{i}:[0, \infty) \rightarrow X$ of the rays $R_{i}$. Denote by $\partial r_{i} \in \partial X$ the corresponding limit points and by $h_{i}$ the corresponding ray horofunctions. Let $G_{i} \subset G$ be the isotropy subgroups of $\partial r_{i}$ and assume that each $G_{i}$ preserves $h_{i}$. Denote by $B_{i}(\rho)$ the open horoballs $h_{i}^{-1}(-\infty, \rho) \subset X$, by $X_{i}(\rho)$ the horospheres $h_{i}^{-1}(\rho)$, and assume that for sufficiently small $\rho$

$$
g B_{i}(\rho) \cap B_{j}(\rho) \neq \emptyset \quad \Longleftrightarrow \quad i=j \text { and } g \in G_{i} .
$$


Let $B(\rho)=\bigcup_{i=1}^{k} B_{i}(\rho)$. Define $X(\rho)=X \backslash G B(\rho)$.

2.2. Definition (Gromov [18, Definition 8.6.A]) A discrete group $G$ is hyperbolic relative to a finite system of subgroups $G_{1}, \ldots, G_{k}$ if $G$ admits an action on some $X$ with the above properties so that $G_{i}$ are the isotropy subgroups of $h_{i}$ and the action of $G$ on $X(\rho)$ is cocompact.

2.3. Assumption. We are going to assume that $X(\rho)$ and the horospheres or strata $X_{i}(\rho)$ are contractible. This makes the quotients $X(\rho) / G$ and $X_{i}(\rho) / G_{i}, 1 \leq i \leq k$, models for finite $B G$ and $B G_{i}$ respectively.

Another assumption which we adopt henceforth that the system $G_{1}, \ldots, G_{k}$ consists of a single subgroup $H$ does not affect the generality of our results and is standard in the reduction theory for lattices.

2.4. Remark. Our definition differs from Gromov's in that we require $X$ to be a $\operatorname{CAT}(\kappa)$ space for some $\kappa<0$ instead of just being hyperbolic. It is not known if this actually narrows the class of groups we handle in this paper (cf. Bridson [2, Introduction]). In any case, all of the known examples of Gromov's relatively hyperbolic groups including the ones in Examples 4 and 5 satisfy our requirement.

2.5. Convention. In the following $\kappa$ will always denote a negative real number.

The Metric in $X(\rho)$. Let $d_{G}$ be the metric in $X(\rho)$ restricted from the hyperbolic metric in $X$. The crucial property of $d_{G}$ is that by choosing a base point $x_{0} \in X(\rho)$ and taking its orbit under the $G$-action we can embed the group $G$ with the word metric quasi-isometrically in $X(\rho)$. This follows from the fact that the hyperbolic $G$-fundamental domains in $X$ restrict to $G$-fundamental domains in $X(\rho)$ which are bounded in this metric.

Geometry of Horospheres. Two sequences $\left\{x_{i}^{1}\right\},\left\{x_{i}^{2}\right\}$ in a metric space $(X, d)$ are called fellow travelers if there is $K>0$ such that $d\left(x_{i}^{1}, x_{i}^{2}\right) \leq K$ for every $i \in \mathrm{N}$.

2.6. Lemma. Let $\left\{y_{i}\right\}$ and $\left\{z_{i}\right\}$ be unbounded sequences in the horospheres $X_{g_{1} H}(\rho)$ and $X_{g_{2} H}(\rho)$ with respect to the metric $d_{G}$. If $g_{1} g_{2}^{-1} \notin H$ then the sequences do not fellow-travel in this metric.

Proof. Consider the closure $\varepsilon X(\rho)$ of $X(\rho)$ in the ideal compactification $\varepsilon X$. Now the cluster points of the two sequences are the distinct ideal points $g_{1} \cdot \partial r$ and $g_{2} \cdot \partial r$; so the given sequences cannot fellow travel in the hyperbolic metric.

2.7. Corollary (Bounded Horosphere Interaction). If $G$ is relatively hy- 
perbolic and $X(\rho)$ is the corresponding stratified space then the horospheres $G X_{H}(\rho)$ satisfy the following property : $g_{1} X_{H}(\rho) \cap N_{d}\left(g_{2} X_{H}(\rho)\right)$ is bounded in $g_{1} X_{H}(\rho)$ for any $d>0$ whenever $g_{1} g_{2}^{-1} \notin H$.

2.8. REMARK. The analogue of this property is a consequence of Farb's Bounded Coset Penetration property in his context.

Visibility in $\mathrm{CAT}(\kappa)$ spaces. Following Eberlein-O'Neill [13] we identify crucial properties of $\mathrm{CAT}(\kappa)$ spaces as

Axiom 1 (Visibility). For any points $c \neq d$ in $\partial X$ there exists at least one geodesic line asymptotic to both $c$ and $d$.

Axiom 2. For any points $c \neq d$ in $\partial X$ there exists at most one geodesic line asymptotic to both $c$ and $d$.

All $\operatorname{CAT}(\kappa)$ spaces are Gromov hyperbolic and so satisfy Axiom 1 according to [15, Proposition 7.1.6].

2.9. Proposition. Each $\mathrm{CAT}(\kappa)$ space X satisfies Axiom 2.

Proof. Given two asymptotic geodesic lines $\gamma, \sigma$ in $X$, let $\gamma(0)=x$, $\sigma(0)=y, \gamma(\infty)=\sigma(\infty)=c$. Consider an increasing sequence $t_{i}>0$ and the corresponding geodesic triangles $\triangle\left(x, y, \gamma\left(t_{i}\right)\right)$ in $X$ where the side $\left[y, \gamma\left(t_{i}\right)\right]$ is the image of the geodesic $\sigma_{i}$. If

$$
y_{i} \stackrel{\text { def }}{=} \sigma_{i}\left(\sigma_{i}^{-1}\left(\gamma\left(t_{i}\right)\right)+t_{0}-t_{i}\right)
$$

then $\lim y_{i}$ is the intersection of $(\sigma)$ and the horosphere centered at $c$ passing through $\gamma\left(t_{0}\right)$. Comparing this picture with the comparison picture in $M_{\kappa}$, one sees that

$$
\lim _{t_{0} \rightarrow \infty} d\left(\gamma\left(t_{0}\right), \lim y_{i}\right)=0
$$

Thus $X$ satisfies

Zero Axiom. For any two geodesics $\gamma$ and $\sigma$ asymptotic to the same $c \in \partial X, d(\gamma, \sigma)=0$.

This implies Axiom 2 just as in [13, Remark 4.12]: let geodesics $\gamma$ and $\sigma$ be asymptotic to two distinct ideal points of $X$. As in any hyperbolic $\operatorname{CAT}(0)$ space, $t \mapsto d(\gamma(t), \sigma)$ is bounded and convex, hence constant. But $d(\gamma(t), \sigma) \rightarrow d(\gamma, \sigma)=0$ as $t \rightarrow \infty$, so $\gamma=\sigma$. 


\section{The Method of Proof}

The proof of the Main Theorem improves the method rather than generalizes results from $[16,17]$. This section recalls the structure of the argument and explains the phenomenon which gets used inductively in $\S 5$.

First we should recall the strategy of Carlsson and Pedersen in [7]. They use control at infinity over certain spaces whose properties are postulated as hypotheses (with emphasized terms explained immediately after the list): the admissible space must (1) be a model for $E G$ with cocompact $G$-action, (2) have a $G$-equivariant compactification $\hat{X}=E G \sqcup Y$, (3) the compactification has to be acyclic with respect to the modified Cech homology with coefficients in $K(R)$, and (4) the boundary $Y$ has to possess a $G$-invariant family $\mathscr{A}$ of boundedly saturated open subsets so that a map defined between the Cech homology spectrum of the one-point compactification $E G^{+}$and the suspension of a similar functor from the coverings by the sets from $\mathscr{A}$ is a weak homotopy equivalence. The conclusion is that the corresponding assembly map $\alpha(G)$ is a split injection.

3.1. Definition. For any subset $K$ of a metric space $(X, d)$ let $K[D]$ denote the set $\{x \in X: d(x, K) \leq D\}$. If $(X, d)$ is embedded in a topological space $\hat{X}$ as an open dense subset, a set $A \subset Y=\hat{X}-X$ is boundedly saturated if for every closed subset $C$ of $\hat{X}$ with $C \cap Y \subset A$, the closure of each $D$-neighborhood of $C \backslash Y$ for $D \geq 0$ satisfies $\overline{(C \backslash Y)[D]} \cap Y \subset A$.

3.2. Definition. The Cech homology of a space $Z$ with coefficients in $S$ is the simplicial spectrum valued functor

$$
\breve{h}(Z ; S)=\underbrace{\operatorname{holim}}_{\operatorname{Cov} Z}\left(N_{-} \wedge S\right),
$$

where $\operatorname{Cov} Z$ is the category of finite rigid open coverings of $Z$ defined in [7]. This is a generalized Steenrod homology theory.

The analogous functor from a $G$-category of finite rigid coverings $A$ of $Y$ by the sets from $\mathscr{A}$ is the homotopy limit of $N A \wedge S$. The map between these functors will be defined shortly.

The idea of proof in [7] is to interpret $\alpha(G)$ as the $G$-fixed point set map between two $G$-spectra

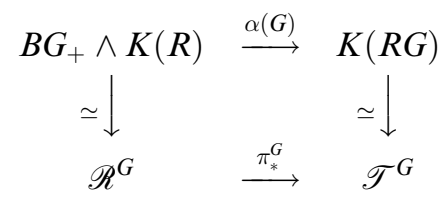


The fixed point set map induces a map on homotopy fixed points and the following commutative diagram:

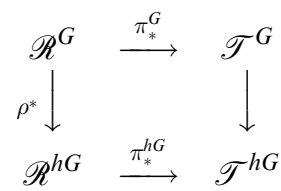

They show that $\rho^{*}: \mathscr{R}^{G} \simeq \mathscr{R}^{h G}$.

The next step is the construction of a new target and a map from $\mathscr{T}$. The target chosen in [7] is $\Sigma N A \wedge K(R)$; the existence of a map from $\mathscr{T}$ is guaranteed by the property of being excisive which all pairs of open sets possess. Now there are equivariant weak homotopy equivalences

$$
\mathscr{R} \longrightarrow \check{h}\left(E G^{+} ; K(R)\right) \longrightarrow \Sigma \underset{\operatorname{Cov} Y}{\operatorname{holim}} N_{-} \wedge K(R)
$$

and a map

$$
\theta: \underbrace{\operatorname{holim}}_{\operatorname{Cov} Y} N_{-} \wedge K(R) \longrightarrow \underbrace{\text { holim }}_{A \in\{A\}} N A \wedge K(R)
$$

such that $\Sigma \theta$ completes the commutative diagram. If $\theta$ is a weak equivalence then the induced map $\mathscr{R}^{h G} \rightarrow(N A \wedge K(R))^{h G}$ is too, and one has $\alpha(G)$ as the first map in a composition which is an equivalence. This makes $\alpha(G)$ a split injection.

When $\mathscr{A}$ are open sets, $\theta$ coincides with the restriction map induced by the inclusion $\{A\} \subset \operatorname{Cov} Y$. This is precisely the map in hypothesis (4) above. In a more general situation when $\mathscr{A}$ are not open but still excisive, one has the same diagram. To identify $\theta$, however, one has to impose the condition that $\mathscr{A}$ is generated as a Boolean algebra from some excisive collection of subsets. Then $\theta$ is induced by a functorial saturation procedure applied to open subsets of $Y$.

There were additional nuances in applying even this generalized scheme to the groups $G$ in [17]. We do not need to repeat the details because they will be essentially incorporated in the inductive assumption we are about to make. Before doing that we simply collect the formal properties of $\mathscr{A}$ that are used in the argument from [17, §9]. They ensure that the analogue of $\theta$ is again a weak equivalence.

3.3. ReQuirements. 1. There is a subcategory Ord $Y$ of $\operatorname{Cov} Y$ such that the inclusion $\jmath$ : Ord $Y \hookrightarrow \operatorname{Cov} Y$ induces a weak homotopy equivalence;

2. For each set $U=\phi(y)$ for $\phi \in \operatorname{Ord} Y$ there is an open set $V(U) \subset \hat{X}$ with the following properties: (1) $V \cap Y=U$ and (2) $\{V(U): U \in \operatorname{im} \phi\}_{\text {Ord } Y}$ form a cofinal system of finite coverings of $Y$ by open subsets of $\hat{X}$; 
3. Given a covering $\phi \in \operatorname{Ord} Y$, there is an assignment (which we call $s a$ turation and denote by sat) of a based boundedly saturated subset $A_{y} \subset Y$ to each set $\phi(y)$ so that sat induces a natural transformation

$$
\mathbf{s a t}_{*}: N_{-} \wedge K(R) \longrightarrow N \operatorname{sat}\left(\_\right) \wedge K(R),
$$

and the collection $\mathscr{A}$ in the previous paragraph is precisely the result of applying saturation to Ord $Y$. We require that each each morphism sat ${ }_{*}$ is a weak equivalence of spectra by Quillen's Theorem A applied to sat $_{*}: N_{-} \rightarrow N$ sat(_).

3.4. Assumption. One can handle $E H=X_{H}(\rho)$ as required by the method producing a proof of the Novikov conjecture for $H$. In particular, there is a compactification $\hat{X}_{H}(\rho)$ of $X_{H}(\rho)$ by the boundary $Y_{H}$ where a collection of boundedly saturated subsets satisfies the requirements above.

3.5. Example (Cusp Subgroups in Hyperbolic Lattices). Choosing $X$ to be the negatively curved symmetric space associated to a semi-simple linear algebraic Q-group of real rank one and $G$ a torsion-free arithmetic subgroup puts us in the situation of [17, Theorem 3]. More generally, one can consider a complete non-compact finite-volume Riemannian manifold $V$ with pinched negative sectional curvatures $-a^{2} \leq K \leq-b^{2}<0$ and torsion-free fundamental group $G=\pi_{1}(V)$. The universal cover $X=\tilde{V}$ satisfies the requirements in Definition 2, so $G$ is hyperbolic relative to the cusp subgroups. As before, we assume that there is a unique cusp of $V$ with the corresponding cusp subgroup $H$. It is known that $H$ is a torsion-free finitely generated nilpotent group. The stratum $X_{H}(\rho)$ can be viewed as the underlying space of a connected simply connected nilpotent Lie group, and $H$ acts on $X_{H}(\rho)$ via the left multiplication action. Since $X_{H}(\rho)$ is contractible, Assumption 2.3 is satisfied. By [17, §4] Assumption 3.3 is also satisfied. Requirements 3 are verified in [17, $\S 5$ and $\S 9.2]$.

\section{Compactification}

According to Assumption 4, we have a compactification $\hat{X}_{H}(\rho)$ with all the required properties. The union of all horospheres $X_{g H}(\rho)=g X_{H}(\rho)$ can be written as the diagonal quotient $X_{H}(\rho) \times_{H} G$. These are the horospheres corresponding to rays $g \cdot r$ from Definition 2.2. Such rays converge to points in $\partial X$ which we call $G$-rational. All the other points in $\partial X$ are called $G$-irrational; they form the subspace $I_{G}$.

4.1. Definition. Let $Y_{H}$ denote the boundary $\hat{X}_{H}(\rho)-X_{H}(\rho)$. Define 


$$
\begin{array}{r}
\hat{X}(\rho) \stackrel{\text { def }}{=} X(\rho) \cup\left(\hat{X}_{H}(\rho) \times_{H} G\right) \cup I_{G}, \\
\delta X(\rho) \stackrel{\text { def }}{=}\left(\hat{X}_{H}(\rho) \times_{H} G\right) \cup I_{G}, \\
Y \stackrel{\text { def }}{=} \hat{X}(\rho)-X(\rho)=\left(Y_{H} \times_{H} G\right) \cup I_{G} .
\end{array}
$$

The topology in $\hat{X}(\rho)$ will be introduced à la Bourbaki. We have in mind

4.2. Proposition ([3, Proposition 1.2.2]). Let $S$ be a set. If to each $x \in S$ there corresponds a set $\mathscr{N}(x)$ of subsets of $S$ such that

1. every subset of $S$ containing one from $\mathscr{N}(x)$ itself belongs to $\mathscr{N}(x)$,

2. a finite intersection of sets from $\mathscr{N}(x)$ belongs to $\mathscr{N}(x)$,

3. the element $x$ belongs to every set in $\mathscr{N}(x)$,

4. for any $N \in \mathscr{N}(x)$ there is $W \in \mathscr{N}(x)$ such that $N \in \mathscr{N}(y)$ for every $y \in W$,

then there is a unique topology on $S$ such that, for each $x \in S, \mathscr{N}(x)$ is the set of neighborhoods of $x$.

By a neighborhood of a subset $A$ in a topological space they understand any subset which contains an open set containing $A$.

The subspace $X(\rho) \subset X$ has the induced topology, so for $y \in X(\rho)$ let $\mathscr{N}(y)=\{\mathcal{O} \subset \hat{X}(\rho): \mathcal{O}$ contains an open neighborhood of $y$ in $X(\rho)\}$.

If $y \in I_{G} \subset \partial X$ then let $\mathscr{N}(y)=\{\mathcal{O} \subset \hat{X}(\rho): W \cap X(\rho) \subset \mathcal{O}$ for some neighborhood $W$ of $y$ in $\varepsilon X$ \}.

4.3. Notation. Given an open subset $U \subset \hat{X}_{g H}(\rho)$, consider all geodesic lines $\ell$ in $X$ which (1) converge to $g \cdot \partial r \in \partial X$ and satisfy (2) $(\ell) \cap U \neq \emptyset$. Let $\mathscr{L}(U)=\bigcup_{\ell} \operatorname{im}(\ell)$ and let $\mathscr{O}(U)=\operatorname{int}(\mathscr{L}(U) \cap X(\rho))$. Now define $\mathscr{C}(U)=$ $\mathcal{O}(U) \cup\left\{z \in Y_{g^{\prime} H}\right.$ : there is $\ell \in \mathscr{L}(U)$ such that $\left.g \cdot \partial r=\lim (\ell)\right\} \cup\left\{z \in I_{G} \subset\right.$ $\partial X$ : there is $\ell \in \mathscr{L}(U)$ such that $z=\lim (\ell)\}$.

Now for $y \in Y_{g H}$, let $\mathscr{N}(y)=\left\{\mathcal{O} \subset \hat{X}(\rho):\right.$ there is an open set $U \subset Y_{g H}$ containing $y$ with $\mathscr{C}(U) \subset \mathcal{O}$.

This defines a system of subsets $\mathscr{N}(y)$ for any $y \in \hat{X}(\rho)$. For a subset $\mathscr{S} \subset \hat{X}(\rho)$ let $\mathscr{N}(\mathscr{S})=\{\mathcal{O} \subset \hat{X}(\rho): \mathcal{O} \in \mathscr{N}(y)$ for every $y \in \mathscr{S}\}$ and call $\mathscr{S}$ open if $\mathscr{S} \in \mathscr{N}(\mathscr{S})$.

4.4. Proposition. If $U \subset \hat{X}_{g H}(\rho)$ is an open subset then $\mathscr{C}(U)$ is open in $\hat{X}(\rho)$.

The proof will rely on the combination of visibility and convexity properties of $\operatorname{CAT}(\kappa)$ spaces. We are greatly aided by the exposition in Bridson [2, $\S \S 1,2]$.

Let $c_{n}$ be a sequence in $\partial X$ coverging to $c$. We will use the notation $c_{p}, c_{n, p}$ for the unique geodesic rays representing these points and based at some $p \in X$. According to [2, Lemma 1.6], $c_{n}$ converge to $c$ if and only if $c_{n, q}$ con- 
verge to $c_{q}$ pointwise for all $q \in X$. Combining this with Proposition 6 one has

4.5. Lemma. Given a geodesic line $\ell$ in a hyperbolic $\mathrm{CAT}(0)$ space $X$ and $a$ sequence $c_{n} \in \partial X$ converging to $\partial_{+} \ell$, there is a sequence of geodesic lines $\ell_{n}$ with $\partial_{-} \ell_{n}=\partial_{-} \ell$ and $\partial_{+} \ell_{n}=c_{n}$ which converges to $\ell$ pointwise.

Proof of Proposition 4.4. We will show that the complement of $\mathscr{C}(U)$ is closed.

If $x \in X(\rho)$ and $x_{n} \notin \mathscr{C}(U)$ is a sequence in $\hat{X}(\rho)$ converging to $x$ then we can assume that $x_{n} \in X(\rho) \subset X$. For the unique rays $\sigma_{n}:\left(-\infty, S_{n}\right] \rightarrow X$ such that $\sigma_{n}\left(S_{n}\right)=x_{n}, \sigma_{n}(0) \in X_{g H}, \partial_{-} \sigma_{n}=g \cdot \partial r$, the unique ray $\sigma:(-\infty, S] \rightarrow X$ with $\sigma(S)=x, \sigma(0) \in X_{g H}, \partial_{-} \sigma=g \cdot \partial r$ is the pointwise limit of $\sigma_{n}$. Thus $\sigma(0)=\lim \sigma_{n}(0) \notin U$ and $x \notin \mathcal{O}(U)$.

If $x \in Y$, consider the corresponding ideal point $c=c(x)$ which is either $g^{\prime} \cdot \partial r$ if $x \in Y_{g^{\prime} H}$ or $x$ itself if $x \in I_{G}$ and a sequence $x_{n}$ in $\partial X$ converging to $c$ so that $(\gamma) \cap U=\emptyset$ for all geodesic lines $\gamma$ with $\partial_{-} \gamma=g \cdot \partial r, \partial_{+} \gamma=x_{n}$. It remains to prove that $(\ell) \cap U=\emptyset$ for each geodesic line $\ell$ with $\partial_{-} \ell=g \cdot \partial r$, $\partial_{+} \ell=c$. Apply Lemma 4.5 to $c_{n}=x_{n}$. The lines $\ell_{n}$ can be parametrized so that $\ell_{n}(0) \in X_{g H}$, hence $\ell(0)=\operatorname{im}(\ell) \cap X_{g H}=\lim \ell_{n}(0) \notin U$.

4.6. Proposition. If $U_{1}, U_{2} \subset \hat{X}_{g H}(\rho)$ are open subsets, then

$$
\mathscr{C}\left(U_{1}\right) \cap \mathscr{C}\left(U_{2}\right)=\mathscr{C}\left(U_{1} \cap U_{2}\right) .
$$

Proof. This follows from Proposition 2.9.

4.7. THEOREM. The open subsets of $\hat{X}(\rho)$ form a well-defined topology in $\hat{X}(\rho)$.

Proof. We need to check that the four characteristic properties from Proposition 2 are satisfied by the system of neighborhoods $\mathscr{N}(x), x \in \hat{X}(\rho)$. (1) and (3) are clear from definitions. (2) follows from Proposition 5. Given any $N \in \mathscr{N}(x), x \in \delta X_{g H}(\rho)$, there is $U \in \hat{X}_{g H}(\rho)$ such that $\mathscr{C}(U) \subset N$. Take $W=\mathscr{C}(U)$. By Proposition 4.4, $N \in \mathscr{N}(y)$ for any $y \in W$. Thus (4) is also satisfied.

This construction properly generalizes that of $\hat{X}$ in $[17, \S 6]$. See [17, Example 6.2.7] for an explicit example.

4.8. TheOREM. The space $\hat{X}(\rho)$ is Hausdorff, compact, and Čech-acyclic.

Proof. This proof uses the same strategy as the proofs in $[17, \S 7]$ and some of the results from that section. In particular, we use the easy fact that the projection $p: \hat{X}(\rho) \rightarrow \varepsilon($ int $X(\rho))$ which collapses each $\hat{X}_{g H}(\rho)$ to an ideal point is continuous. 
Given two points $x_{1}, x_{2} \in \hat{X}(\rho)$ with $p\left(x_{1}\right) \neq p\left(x_{2}\right)$, for any two neighborhoods $U_{1}, U_{2} \subset \varepsilon($ int $X(\rho))$ of $x_{1}$ and $x_{2}$ respectively, if $U_{1} \cap U_{2}=\emptyset$ then $p^{-1}\left(U_{1}\right) \cap p^{-1}\left(U_{2}\right)=\emptyset$. Since $\varepsilon($ int $X(\rho))$ is Hausdorff, one is always able to separate such $x_{1}$ and $x_{2}$. If $p\left(x_{1}\right)=p\left(x_{2}\right)$ and $x_{1} \neq x_{2}$ then $x_{1}, x_{2} \in \hat{X}_{g H}(\rho)$ for some $g \in G$. Using the assumption that $\hat{X}_{g H}(\rho)$ is Hausdorff, let $V_{1}$ and $V_{2}$ be open neighborhoods of $x_{1}$ and $x_{2}$ with $V_{1} \cap V_{2}=\emptyset$. Then $\mathscr{C}\left(V_{1}\right) \cap \mathscr{C}\left(V_{2}\right)=\emptyset$. This proves the Hausdorff property.

Compactness follows as in [17, §7.3] from Proposition 4.6 and compactness of the ideal compactification $\varepsilon($ int $X(\rho))$ and of $\hat{X}_{H}(\rho)$.

There is a weak Vietoris-Begle theorem for the modified Cech homology [17, Theorem 7.4.1]. The fibers need only be acyclic for the result of Inassaridze used in the proof of that theorem, so if $f: X \rightarrow Y$ is a surjective continuous map, where $Y$ is contractible and $f^{-1}(y)$ are acyclic for all $y \in Y$, then

$$
f_{*}: \check{h}(X ; K R) \longrightarrow \check{h}(Y ; K R)
$$

is a weak homotopy equivalence. Now the ideal compactification $\varepsilon(X)$ is contractible for any $\operatorname{CAT}(0)$ space $X$, so $\varepsilon($ int $X(\rho))$ homotopy equivalent to $\varepsilon(X)$ is contractible. The fibers of $p$ are either assumed to be acyclic or consist of single points. This shows Čech-acyclicity of $\hat{X}(\rho)$.

\section{Proof of the Main Theorem}

Bounded Saturation in $Y_{G}$. We will use the geometry of $Y$ to identify a sufficiently fine collection of subsets which are boundedly saturated in the sense of Definition 3.1.

5.1. Proposition. Each G-irrational point $y \in I_{G} \subset Y$ is boundedly saturated. If a subset $\Omega \subset Y_{g H}$ is boundedly saturated with respect to the metric in $X_{g H}(\rho)$ then it is also boundedly saturated with respect to the metric $d_{G}$ in $X(\rho)$.

Proof. First, let $y \in I_{G}$. Consider any closed subset $C \subset \hat{X}(\rho)$ with $C \cap Y_{G}=\{y\}$. We can without a loss of generality assume that $\overline{C \backslash Y_{G}} \cap Y_{G}=\{y\}$ so that $y$ is the unique cluster point in $\partial X$ of any unbounded sequence in $C$. Suppose that $z \in \overline{\left(C \backslash Y_{G}\right)[D]} \cap Y_{G}$. Then there is an unbounded sequence in $\left(C \backslash Y_{G}\right)[D]$ converging to $z$ which must be a fellowtraveler of a sequence converging to $y$. If $z \notin I_{G}$ then any sequence converging to $z$ converges to $\partial r_{g H} \neq y$ in $\partial X$. Finally, if $z \in I_{G}$ then $z=y$.

Suppose $\Omega \subset Y_{g H}$ is boundedly saturated with respect to $d \mid X_{g H}(\rho)$. Consider a closed set $C$ with $\overline{C \backslash Y_{G}} \cap Y_{G} \subset \Omega$. By the argument above and Cor- 
ollary 2.7, $\quad \overline{\left(C \backslash Y_{G}\right)[D]} \cap \partial X=\partial r_{g H} . \quad$ So $\quad \overline{\left(C \backslash Y_{G}\right)[D]} \cap \delta X \subset \hat{X}_{g H} \quad$ and $\overline{\left(C \backslash Y_{G}\right)[D]} \cap \partial X_{g H} \subset \Omega$.

The rest of the proof uses the same idea and constructions as in $[17, \S 9]$ the latter becoming more formal in view of the formal requirements isolated in 3.3 .

Orderly Coverings. We assume that the boundary $Y_{g H}$ of a stratum $X_{g H}(\rho)$ guaranteed by Assumption 3.4 satisfies all of Requirements 3.3. So there is a category of rigid coverings $\operatorname{Ord} Y_{g H}$ and open subsets $V\left(U^{\psi}(y)\right) \subset \hat{X}_{g H}(\rho)$ for each $y \in Y_{g H}$ and $\psi \in \operatorname{Ord} Y_{g H}$ as in 3.3(2). Notice that by the definition of topology in $\hat{X}$, for any $\mathscr{U} \in \operatorname{Cov} \hat{X}$, there is $\psi \in \operatorname{Ord} Y_{g H}$ such that the influx sets $\mathscr{C}\left(V\left(U^{\psi}(y)\right)\right)$ refine the covering $\mathscr{U}(y)$ for $y \in Y_{g H}$. We will denote them by $\mathscr{C}\left(U^{\psi}(y)\right)$. Consider

$$
\operatorname{ExcOrd}^{\psi}(y)=Y \cap \mathscr{C}\left(U^{\psi}(y)\right) \backslash \bigcup_{g^{\prime} \in \mathscr{G}} Y_{g^{\prime} H},
$$

where $\mathscr{G}$ is the set of all $g^{\prime}$ with $Y_{g H} \not \subset \mathscr{C}\left(U^{\psi}(y)\right)$, and define

$$
\operatorname{Ord}^{\psi}(y)=\operatorname{ExcOrd}^{\psi}(y) \cup V\left(U^{\psi}(y)\right) .
$$

If $y \in I_{G}$, let $U$ be an open neighborhood of $y$ in $\hat{X}$ and define

$$
\operatorname{Ord}^{U}(y)=Y \cap U \backslash \bigcup_{g^{\prime} \in \mathscr{G}} Y_{g^{\prime} H},
$$

where $\mathscr{G}$ is the set of all $g^{\prime}$ with $Y_{g H} \not \subset U$. These sets are open neighborhoods of $y$.

By compactness of $\hat{X}$, for any $\mathscr{U} \in \operatorname{Cov} \hat{X}$ there are finite collections $\mathscr{B}_{G} \subset G$ and $\mathscr{B}_{I} \subset I_{G}$ with some choices of $\psi_{g}$ in $\hat{X}_{g H}(\rho)$ for $g \in \mathscr{B}_{G}$ and $U_{y}$ for $y \in \mathscr{B}_{I}$ so that $\left\{\operatorname{Ord}^{\psi_{g}}(z), \operatorname{Ord}^{U}(y) \mid z \in Y_{g H}, g \in \mathscr{B}_{G}, y \in \mathscr{B}_{I}\right\}$ is a finite covering of $\hat{X}$ which refines the covering by open sets from $\mathscr{U}$. We will also want to allow the sets $\operatorname{ExcOrd}^{\psi_{g}}(z) \subset \operatorname{Ord}^{\psi_{g}}(z)$ to be included in this covering; this certainly does not affect the cofinality property mentioned above.

5.2. Definition. The category PreOrd $Y$ consists of rigid open coverings $\beta$ such that

1. im $\beta$ is an open covering of $Y$ constructed as above,

2. $y \in Y_{g H}$ for some $g \in \mathscr{B}_{G} \Longleftrightarrow \beta(y)=\operatorname{Ord}^{\psi_{g}}(z)$ for some $z \in Y_{g H}$.

Define Ord $Y$ to be the full subcategory of Cov $Y$ closed under rigid intersections of covering sets generated by PreOrd $Y$.

It is easy to see that Ord $Y$ is not left cofinal in Cov $Y$ but satisfies the hypotheses on the category $\mathscr{C}$ in $[17, \S 2.4]$. The conclusion of that section was that the map 


$$
J^{*}: \check{h}(Y ; K R) \longrightarrow \underbrace{\operatorname{holim}}_{\operatorname{Ord} Y}\left(N_{-} \wedge K R\right)
$$

induced by the inclusion $\mathrm{g}$ : Ord $Y \hookrightarrow \operatorname{Cov} Y$ is a weak homotopy equivalence.

According to Requirement 3.3(3), each $Y_{g H}, g \in G$, has an assignment sat associated to each $\psi \in$ Ord $Y$.

5.3. Definition. Given a covering $\omega \in \operatorname{Ord} Y$, let sat $(\omega)$ be the covering by boundedly saturated oversets obtained from $\omega$ by applying sat in each $Y_{g H}$. Now we let $\{A\}$ consist of all finite rigid coverings obtained from Ord $Y$ by applying saturation.

Completion of the Proof. Inclusions of nerves $N(\omega) \hookrightarrow N \operatorname{sat}(\omega)$ induce natural weak equivalences $N(\omega) \wedge K R \simeq N \operatorname{sat}(\omega) \wedge K R$ by Quillen's Theorem A. This follows from the fact that factoring out a contractible subcomplex generated by a subset of vertices factors through the inclusion into the complex where the same subset generates a simplex. This is precisely what happens with finitely many disjoint subcomplexes associated to sets covering $Y_{g H}, g \in \mathscr{B}_{G}$, using property (2) from Definition 5.2. We can conclude that $\alpha_{*}$ is a weak equivalence by the homotopy invariance of homotopy inverse limits.

5.4. Example (Pinched Hyperbolic Manifolds). This example was the motivation for introducing relatively hyperbolic spaces and groups. The constructions in Definition 2.2 are classical for these manifolds. The manifold with boundary $X(\rho)$ is homotopy equivalent to its interior which is contractible. It is known that the cusp subgroups in a complete non-compact finite-volume Riemannian manifold with pinched negative sectional curvatures are torsion-free, finitely generated, and nilpotent. Example 3.5 applies verbatim. Thus Assumptions 2.3 and 3.4 hold for these spaces and the Main Theorem applies to the fundamental groups.

5.5. Example (Relative Strict Hyperbolization). Strict hyperbolization is a procedure for converting an arbitrary polyhedron into another polyhedron with curvature bounded above by some negative number. A version of such process is provided by [10]. The first step in the process is asphericalization which results in a polyhedron with a piecewise Euclidean metric of non-positive curvature. It is naturally a cubical cell complex with each cell isometric to a standard Euclidean cube. This step has been described first in [18] and given careful treatment by Davis-Januszkiewicz $[11,20]$ and F. Paulin. There existed a hope that a perturbation of this new metric would make the curvature strictly negative. This turned out to be false, but the work of Charney-Davis [10] describes the second step necessary to make the hy- 
perbolization strict. They replace each cube-face with a real rank one arithmetic quotient (of negative curvature). The resulting space has curvature bounded above by -1 . The trade-off is the loss of control over the topology of the space. In particular, we do not understand which hyperbolic groups appear as the fundamental groups of the resulting hyperbolic space and how they are related to the fundamental group of the original polyhedron.

Now we wish to describe a procedure which might be called relative strict hyperbolization and is suggested by [20, p. 472-473]. There are various conditions that are reasonable to impose on a construction if it is to bear such a nameÅsee [10, p. 335]. Here our only concern is production of relatively hyperbolic groups satisfying Assumptions 2.3 and 3.4, and this procedure will suffice.

Let $L$ be a finite simplicial complex which is a classifying space for the fundamental group $H$ such that the universal cover $E H$ satisfies Assumption 3.4. This means that the method for proving the Novikov conjecture for $H$ described in $\S 3$ can be applied to $L$. So $H$ might be a torsion-free finitely generated nilpotent group or a relatively hyperbolic group for which the Main Theorem is known to be true. Embed $L$ in an arbitrary finite simplicial complex $K$. Consider the cone of the inclusion $L \hookrightarrow K$ and perform the strict hyperbolization of the resulting complex. Call the result $V$. The normal link of the cone point is PL-homeomorphic to $L$. Excising the regular neighborhood of the cone point, one gets a compact complex which is a classifying space by Vietoris-Begle. Let $G$ be the fundamental group of this space. Now the universal cover $X$ of $V$ and the group $G$ satisfy the requirements of Definition 2.2 making $G$ hyperbolic relative to $H$. Assumptions 2.3 and 3.4 are satisfied by default, so the Main Theorem applies to $G$.

\section{REFERENCES}

1. J. Alonso and M. Bridson, Semihyperbolic groups, Proc. London Math. Soc. 70 (1995), 56114.

2. M. Bridson, On the existence of flat planes in spaces of nonpositive curvature, Proc. Amer. Math. Soc. 123 (1995), 223-235.

3. N. Bourbaki, General topology. Chapters 1-4, Springer (1989).

4. G. Carlsson, Homotopy fixed points in algebraic K-theory of certain infinite discrete groups, in Advances in homotopy theory (S.M. Salamon, B. Steer, and W.A. Sutherland, eds.), Cambridge U. Press (1989), 5-10.

5. G. Carlsson, Bounded K-theory and the assembly map in algebraic K-theory, in Novikov conjectures, index theory and rigidity, Vol. 2 (S.C. Ferry, A. Ranicki, and J. Rosenberg, eds.), Cambridge U. Press (1995), 5-127.

6. G. Carlsson and E.K. Pedersen, Controlled algebra and the Novikov conjectures for $K$ - and Ltheory, Topology 34 (1993), 731-758. 
7. G. Carlsson and E.K. Pedersen, Čech homology and the Novikov conjectures for K- and Ltheory, Math. Scand. 82 (1998), 5-47.

8. G. Carlsson, E.K. Pedersen, and W. Vogell, Continuously controlled algebraic K-theory of spaces and the Novikov conjecture, Math. Ann. (1997).

9. G. Carlsson, E.K. Pedersen, and J. Roe, Controlled $C^{*}$-algebra theory and the injectivity of the Baum-Connes map, in preparation (1996).

10. R. Charney and M. Davis, Strict hyperbolization, Topology 34 (1995), 329-350.

11. M. Davis and T. Januszkiewicz, Hyperbolization of polyhedra, J. Differential Geom. 34 (1991), 347-388.

12. P. Eberlein, Lattices in spaces of nonpositive curvature, Ann. of Math. 111 (1980), 435-476.

13. P. Eberlein and B. O’Neill, Visibility manifolds, Pacific J. Math. 46 (1973), 45-109.

14. B. Farb, Relatively hyperbolic and automatic groups with applications to negatively curved manifolds, Ph.D. thesis, Princeton U. (1994).

15. E. Ghys and P. de la Harpe, eds., Sur les groupes hyperboliques d'après Mikhael Gromov, Prog. Math. 83, 1990.

16. B. Goldfarb, Splitting assembly maps for arithmetic groups with large actions at infinity, Ph.D. thesis, Cornell U. (1996).

17. B. Goldfarb, Novikov conjectures for arithmetic groups with large actions at infinity, $K$-theory 11 (1997).

18. M. Gromov, Hyperbolic groups, in Essays in group theory (S.M. Gersten, ed.), M.S.R.I. Publication 8 (1987), 75-264.

19. M. Gromov and W. Thurston, Pinching constants for hyperbolic manifolds, Invent. Math. 89 (1987), 1-12.

20. T. Januszkiewicz, Hyperbolizations, in Group theory from a geometric viewpoint (E. Ghys, A. Haefliger, and A. Verjovsky, eds.), World Scientific (1991), 464-490. 